\title{
Closed Orbit Response to Quadrupole Strength Variation
}

\author{
A. Wolski \\ Ernest Orlando Lawrence Berkeley National Laboratory, Berkeley, CA 94720 \\ F. Zimmermann \\ CERN
}

January $20^{\text {th }}, 2004$

\begin{abstract}
We derive two formulae relating the variation in closed orbit in a storage ring to variations in quadrupole strength, neglecting nonlinear and dispersive effects. These formulae correct results previously reported $[1,2,3]$. We compare the results of the formulae applied to the ATF with simulations using MAD, and consider their application to beam-based alignment.
\end{abstract}

\section{Disclaimer}

This document was prepared as an account of work sponsored by the United States Government. While this document is believed to contain correct information, neither the United States Government nor any agency thereof, nor The Regents of the University of California, nor any of their employees, makes any warranty, express or implied, or assumes any legal responsibility for the accuracy, completeness, or usefulness of any information, apparatus, product, or process disclosed, or represents that its use would not infringe privately owned rights. Reference herein to any specific commercial product, process, or service by its trade name, trademark, manufacturer, or otherwise, does not necessarily constitute or imply its endorsement, recommendation, or favoring by the United States Government or any agency thereof, or The Regents of the University of California. The views and opinions of authors expressed herein do not necessarily state or reflect those of the United States Government or any agency thereof or The Regents of the University of California.

LBNL is an equal opportunities employer.

This work was supported by the Director, Office of Science, of the U.S. Department of Energy under Contract No. DE-AC03-76SF00098. 


\section{Introduction}

Beam-based alignment techniques in storage rings commonly use the closed orbit change with respect to variation of quadrupole strength to determine the beam position with respect to the center of the quadrupole. In many cases, one simply steers the beam until there is no response to the change in quadrupole strength, in which case the beam must be passing through the magnetic center of the quadrupole. Such "nulling" techniques have been applied, for example, at the ALS [1] and the ATF [4]. Typically, one determines the reading on a BPM adjacent to the quadrupole at the point where the beam passes through the center of the quadrupole, then uses the set of BPM readings for all quadrupoles to define a "golden orbit", ideally passing through the center of every quadrupole. (We note that often, and certainly at the ATF, a significant source of the apparent quadrupole to BPM offset arises in the BPM electronics, rather than in a physical relative displacement between the quadrupole and the BPM.) In some cases, the nearest BPM may be some distance from the quadrupole. In such situations, it is useful to know the beam offset with respect to the center of the quadrupole for a given orbit: the ability to determine the offset suggests a variation on the above procedure for defining the golden orbit, which we discuss below.

In practice, there may be insufficient steering magnets to control the beam so that it passes through the center of every quadrupole. At the ATF, for example, there are three quadrupoles (including the gradient bending magnet) per arc cell, but only two BPMs and one horizontal corrector magnet and one vertical corrector magnet per arc cell. Even if it is not possible to steer a completely flat orbit, it may be useful to include known quadrupole offsets in a lattice modeling code, to give a more reliable prediction of the performance of the machine. It may also be possible to identify quadrupoles with particularly large offsets, so that they may be mechanically realigned.

To determine the beam offset with respect to the center of the quadrupole, without having a BPM placed conveniently adjacent to the quadrupole, we can again look at the closed orbit variation with respect to changes in the quadrupole strength. But instead of steering the beam until there is no response to the change in strength, we need to know how the orbit changes with respect to the quadrupole strength for a given initial offset of the beam with respect to the quadrupole center. This is a less straightforward problem than might at first appear: we believe that the formula reported in references [1] and [2], and reproduced in [3], is not correct.

Here, we aim to derive in as clear a manner as possible, two formulae relating the change in closed orbit in a storage ring to the change in strength of a quadrupole. We shall see that the different formulae have different advantages. To test our results, we compare with simulations in MAD.

\section{Deriving the Formulae}

\subsection{Matrix Method}

Consider a quadrupole of integrated strength $K$ in a storage ring. Our convention shall be that a positive value of $K$ means that the quadrupole is horizontally focusing. We shall consider only the horizontal plane, and neglect the effects of dispersion. Let $\mathbf{M}$ be the single-turn linear 
map at the exit of the quadrupole. The map from the exit of the quadrupole around the ring, to the entrance of the same quadrupole is then:

$$
\hat{\mathbf{M}}=\mathbf{M}_{K}^{-1} \cdot \mathbf{M}
$$

where $\mathbf{M}_{K}$ is the map through the quadrupole. For a thin quadrupole:

$$
\mathbf{M}_{K}=\left(\begin{array}{cc}
1 & 0 \\
-K & 1
\end{array}\right)
$$

Let $\left(x_{c o}, p_{c o}\right)$ be the phase space co-ordinates of a particle on the closed orbit at the entrance to the quadrupole; $p$ is effectively the angle of the orbit $x^{\prime}$ with respect to the design orbit. Now we suppose that the closed orbit passes through the quadrupole a distance $x_{b q}$ from the quadrupole center: i.e. $x_{b q}$ is the position of the beam with respect to the quadrupole. The effect on our chosen particle of passing through the quadrupole is the transformation:

$$
\left(\begin{array}{c}
x_{c o} \\
p_{c o}
\end{array}\right) \rightarrow\left(\begin{array}{c}
x_{c o} \\
p_{c o}-K x_{b q}
\end{array}\right)
$$

Now applying the map from the exit of the quadrupole around the ring and back to the entrance of the quadrupole must by definition put us back where we started:

$$
\hat{\mathbf{M}} \cdot\left(\begin{array}{c}
x_{c o} \\
p_{c o}-K x_{b q}
\end{array}\right)=\left(\begin{array}{c}
x_{c o} \\
p_{c o}
\end{array}\right)
$$

Therefore:

$$
\mathbf{M}_{K}^{-1} \cdot \mathbf{M} \cdot\left(\begin{array}{c}
x_{c o} \\
p_{c o}-K x_{b q}
\end{array}\right)=\left(\begin{array}{c}
x_{c o} \\
p_{c o}
\end{array}\right)
$$

Now, we note that $\hat{\mathbf{M}}$ is independent of the quadrupole strength. This means we can write equation (1) for a new quadrupole strength $K^{(1)}$ :

$$
\hat{\mathbf{M}} \cdot\left(\begin{array}{c}
x_{c o}^{(1)} \\
p_{c o}^{(1)}-K^{(1)} x_{b q}^{(1)}
\end{array}\right)=\left(\begin{array}{c}
x_{c o}^{(1)} \\
p_{c o}^{(1)}
\end{array}\right)
$$

where:

$$
\begin{aligned}
& x_{c o}^{(1)}=x_{c o}+\Delta x_{c o} \\
& x_{b q}^{(1)}=x_{b q}+\Delta x_{c o} \\
& p_{c o}^{(1)}=p_{c o}+\Delta p_{c o}
\end{aligned}
$$

Using these definitions, and subtracting equation (1) from equation (2) gives:

$$
\left(\begin{array}{c}
\Delta x_{c o} \\
\Delta p_{c o}
\end{array}\right)=\mathbf{M}_{K}^{-1} \cdot \mathbf{M} \cdot\left(\begin{array}{c}
\Delta x_{c o} \\
\Delta p_{c o}-\Delta K x_{b q}-K^{(1)} \Delta x_{c o}
\end{array}\right)
$$


where

$$
\Delta K=K^{(1)}-K
$$

Now the single-turn map can be written:

$$
\mathbf{M}=\left(\begin{array}{cc}
\cos (2 \pi v)+\alpha \sin (2 \pi v) & \beta \sin (2 \pi v) \\
-\gamma \sin (2 \pi v) & \cos (2 \pi v)-\alpha \sin (2 \pi v)
\end{array}\right)
$$

and equation (3) is then solved to give:

$$
\begin{aligned}
& \Delta x_{c o}=-x_{b q} \frac{\beta \Delta K \cot (\pi v)}{2+\beta \Delta K \cot (\pi v)} \\
& \Delta p_{c o}=x_{b q} \Delta K \frac{1-(\beta K-\alpha) \cot (\pi v)}{2+\beta \Delta K \cot (\pi \nu)}
\end{aligned}
$$

Note: equations (5) give the change in the closed orbit at the entrance to the thin quadrupole, while the Twiss parameters refer to the exit of the quadrupole. Using the map through the quadrupole, it is easy to write down the change in the closed orbit at the exit of the quadrupole:

$$
\begin{aligned}
& \left.\Delta x_{c o}\right|_{\text {exit }}=-x_{b q} \frac{\beta \Delta K \cot (\pi v)}{2+\beta \Delta K \cot (\pi v)} \\
& \left.\Delta p_{c o}\right|_{\text {exit }}=-x_{b q} \frac{\Delta K[1-\alpha \cot (\pi v)]}{2+\beta \Delta K \cot (\pi v)}
\end{aligned}
$$

Equations (6) are the expressions we were seeking, relating the change in the closed orbit at a quadrupole with respect to the change in strength of the quadrupole. They are in a convenient form, in that all quantities are specified at the original strength of the quadrupole. Although they give the change in closed orbit only at one point in the ring (i.e. at the quadrupole) changes in the orbit at other locations may be found simply by applying the transfer map from the exit of the quadrupole to the desired location: this map is of course independent of the quadrupole strength.

\subsection{Quadrupole-Shift Method}

An alternative approach to the problem leads to an expression for the change in orbit resulting from a change in quadrupole strength that looks rather different from equation (6). We shall see in the following section that the two expressions give identical results.

Consider, as before, a quadrupole of integrated strength $K$ with the closed orbit passing a distance $x_{b q}$ from the center of the quadrupole. Moving the quadrupole a distance $\Delta x_{q}$ is physically the same as superposing a dipole field giving a kick to the beam of angle

$$
\theta=K \Delta x_{q}
$$

The closed orbit response at a location $s$ to a kick of angle $\theta$ at location $s_{0}$ is given in terms of components of the closed orbit response matrix $\mathbf{C}$ :

$$
\begin{aligned}
& \Delta x_{c o}(s)=C_{12}\left(s ; s_{0}\right) \theta \\
& \Delta p_{c o}(s)=C_{22}\left(s ; s_{0}\right) \theta
\end{aligned}
$$


Now suppose we move the quadrupole a distance such that the closed orbit now goes through the center of the quad. What would this transverse distance have to be? We can find it from:

$$
\Delta x_{c o}-\Delta x_{q}=-x_{b q}
$$

where if the position is not explicitly specified, $\Delta x_{c o}=\Delta x_{c o}\left(s_{0}\right)$. This gives:

$$
\Delta x_{q}=\Delta x_{c o}+x_{b q}=C_{12}\left(s_{0} ; s_{0}\right) K \Delta x_{q}+x_{b q}
$$

and hence:

$$
\Delta x_{q}=\frac{x_{b q}}{1-C_{12}\left(s_{0} ; s_{0}\right) K}
$$

The change in the closed orbit is:

$$
\begin{aligned}
& \Delta x_{c o}^{(i)}(s)=x_{b q} \frac{C_{12}\left(s ; s_{0}\right) K}{1-C_{12}\left(s_{0} ; s_{0}\right) K} \\
& \Delta p_{c o}^{(i)}(s)=x_{b q} \frac{C_{22}\left(s ; s_{0}\right) K}{1-C_{12}\left(s_{0} ; s_{0}\right) K}
\end{aligned}
$$

Having moved the quadrupole this exact distance, we can now change its strength with impunity: there is no effect on the closed orbit, since the closed orbit goes exactly through the center of the quadrupole. But now we move the quadrupole back to where it started. The closed orbit does not go back to where it started, since the quadrupole strength is now different. Suppose the new quadrupole strength is $K^{(1)}$. The change in closed orbit resulting only from the second move of the quad (back to its original position) is:

$$
\begin{aligned}
\Delta x_{c o}^{(i i)}(s) & =-x_{b q} \frac{C_{12}^{(1)}\left(s ; s_{0}\right) K^{(1)}}{1-C_{12}\left(s_{0} ; s_{0}\right) K} \\
\Delta p_{c o}^{(i i)}(s) & =-x_{b q} \frac{C_{22}^{(1)}\left(s ; s_{0}\right) K^{(1)}}{1-C_{12}\left(s_{0} ; s_{0}\right) K}
\end{aligned}
$$

Note that the closed orbit response matrix $\mathbf{C}$ has changed as a result of the change in the quadrupole strength, and is now denoted $\mathbf{C}^{(1)}$. The total change in the closed orbit is given by the sum of equations (7) and (8):

$$
\begin{aligned}
& \Delta x_{c o}(s)=-x_{b q} \frac{C_{12}^{(1)}\left(s ; s_{0}\right) K^{(1)}-C_{12}\left(s ; s_{0}\right) K}{1-C_{12}\left(s_{0} ; s_{0}\right) K} \\
& \Delta p_{c o}(s)=-x_{b q} \frac{C_{22}^{(1)}\left(s ; s_{0}\right) K^{(1)}-C_{22}\left(s ; s_{0}\right) K}{1-C_{12}\left(s_{0} ; s_{0}\right) K}
\end{aligned}
$$

Equations (9) are the expressions we were seeking. Explicit expressions for the components of the $\mathbf{C}$ matrix are standard: 


$$
\begin{aligned}
& C_{12}\left(s ; s_{0}\right)=\frac{\sqrt{\beta(s) \beta\left(s_{0}\right)}}{2 \sin (\pi v)} \cos \left(\pi v-\left|\psi(s)-\psi\left(s_{0}\right)\right|\right) \\
& C_{22}\left(s ; s_{0}\right)=\frac{1}{2 \sin (\pi v)}\left[\sin \left(\pi v-\left|\psi(s)-\psi\left(s_{0}\right)\right|\right)-\sqrt{\alpha(s) \alpha\left(s_{0}\right)} \cos \left(\pi v-\left|\psi(s)-\psi\left(s_{0}\right)\right|\right)\right]
\end{aligned}
$$

Let us consider the special case of the closed orbit response at the exit of the quadrupole. Then we have:

$$
\begin{aligned}
C_{12} & =\frac{\beta}{2 \sin (\pi v)} \cos (\pi v) \\
C_{22} & =\frac{1}{2 \sin (\pi v)}[\sin (\pi v)-\alpha \cos (\pi v)]
\end{aligned}
$$

and equations (9) become:

$$
\begin{aligned}
& \Delta x_{c o}=-x_{b q} \frac{\beta_{1} K^{(1)} \cot \left(\pi v_{1}\right)-\beta K \cot (\pi \nu)}{2-\beta K \cot (\pi \nu)} \\
& \Delta p_{c o}=-x_{b q} \frac{\left[1-\alpha_{1} \cot \left(\pi v_{1}\right)\right] K^{(1)}-[1-\alpha \cot (\pi \nu)] K}{2-\beta K \cot (\pi \nu)}
\end{aligned}
$$

\subsection{Equivalence of Equations (6) and (10)}

Equations (6) and equations (10) look very different, but with some algebra, we can show they are the same. We shall give the proof only for the first in each set, for the change in coordinate rather than the change in momentum. Begin by considering the effect of a focusing error $\Delta K$, which has the transfer matrix:

$$
\mathbf{M}_{\Delta K}=\left(\begin{array}{cc}
1 & 0 \\
-\Delta K & 1
\end{array}\right)
$$

Multiplying this by the single-turn matrix in the form (4) gives the single-turn matrix in the presence of the focusing error, and from this new matrix we find the following expressions for the new beta function and tune:

$$
\begin{aligned}
\beta_{1} \sin \left(2 \pi v_{1}\right) & =\beta \sin (2 \pi v) \\
\cos \left(2 \pi v_{1}\right) & =\cos (2 \pi v)-\frac{1}{2} \beta \Delta K \sin (2 \pi v)
\end{aligned}
$$

Expanding the trigonometric functions and dividing the first equation by the second gives:

$$
\beta_{1} \cot \left(\pi v_{1}\right)=\frac{\beta \cot (\pi v)}{1+\frac{1}{2} \beta \Delta K \cot (\pi v)}
$$

Now we use this last equation to substitute for $\beta_{1} \cot \left(\pi \nu_{1}\right)$ in the first of equations (10), and also substitute for $K^{(1)}=K+\Delta K$. After a few lines of algebra, the first of equations (5) follows. We note that in applying equations (10) it is important to include the effects of the 
change in quadrupole strength on the beta function and tune; only then will the correct results, equivalent to equations (5), be obtained.

\section{Comparison with Simulations}

It is straightforward to use MAD to model the change in closed orbit resulting from a change in quadrupole strength. Here, we present the results of a simulation using a version of the ATF lattice. We turn off the sextupoles, so that there are no variations in focusing with orbit. We also turn off the RF, so that there are no dispersive effects resulting from a change in path length with distortion of the orbit. In the above analysis we neglected both sextupole and RF effects; for small orbit distortions, these effects should be small, but careful studies should be carried out before applying the results of our analysis in practice. To improve further the correspondence between the simulation and the models we have considered in the above analysis, we use thin quadrupoles.

To further enlighten the reader, we now work in the vertical plane. This means that the quadrupole strength we use in our analytical expressions has to be the negative of the quadrupole strength used in the lattice definition in MAD. This is because we have assumed that a positive $K$ means that the quadrupole is focusing in the plane under consideration; in MAD, a positive $K$ means that the quadrupole is defocusing vertically.

\subsection{Quadrupole QF2R.26}

The QF2R quadrupoles in the ATF are the horizontally focusing quadrupoles in the arcs. For the simulations, we displaced this quadrupole $3 \mathrm{~mm}$ vertically. The relevant parameters as functions of the integrated strength of the quadrupole are given in Table 1. Note that the orbit values are with respect to the reference trajectory. The nominal integrated strength of QF2R.26 is $1.64225 \mathrm{~m}^{-1}$. Changes in the closed orbit with respect to the orbit at the nominal quad strength are plotted in Figure 1 and Figure 2. Also plotted are the changes in the closed orbit calculated from equations (6) and (10); the analytical formulae are in agreement with the simulation results to numerical precision. 


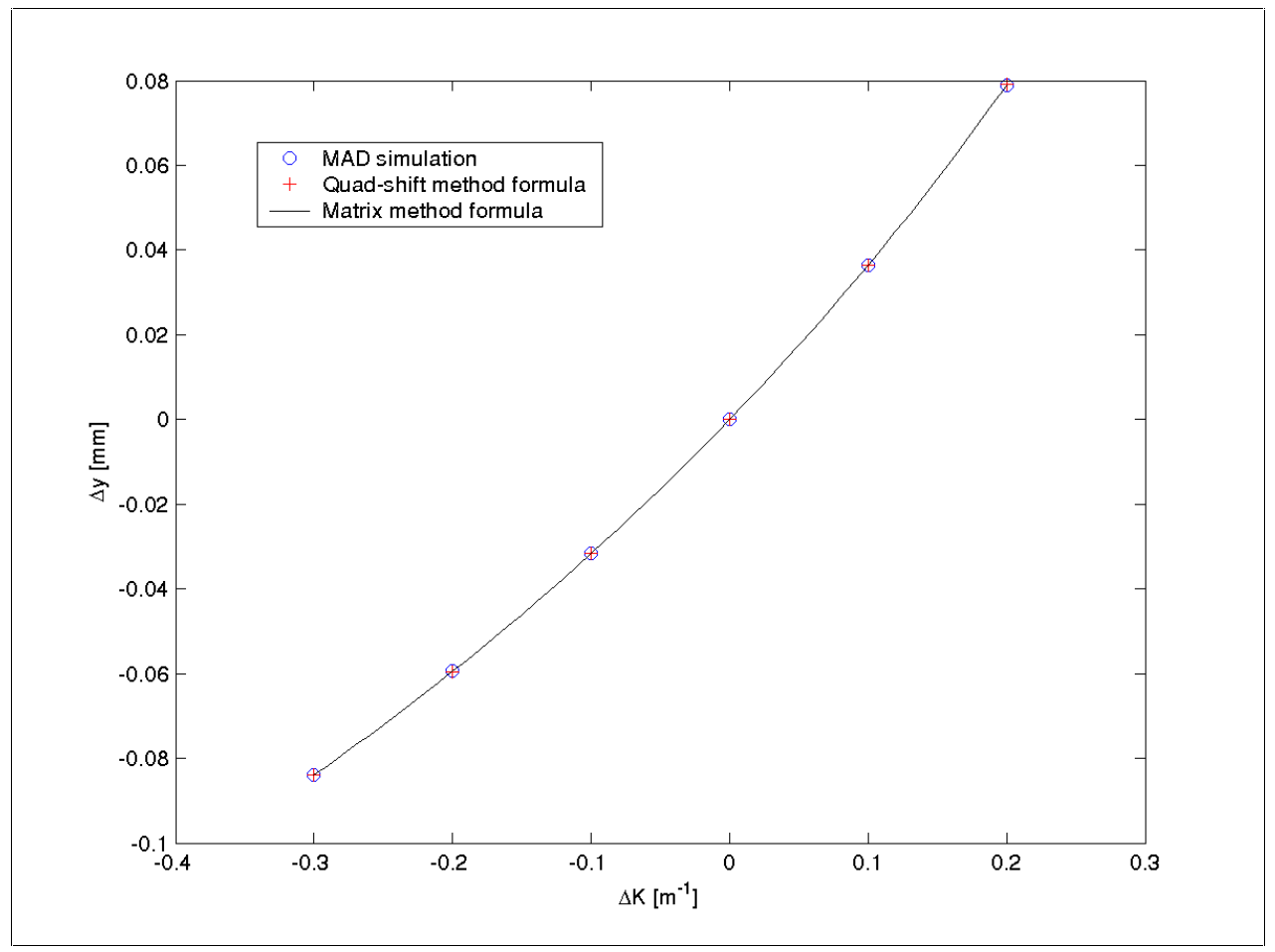

Figure 1

Change of closed orbit co-ordinate at QF2R.26 in response to variations in the strength of the quadrupole.

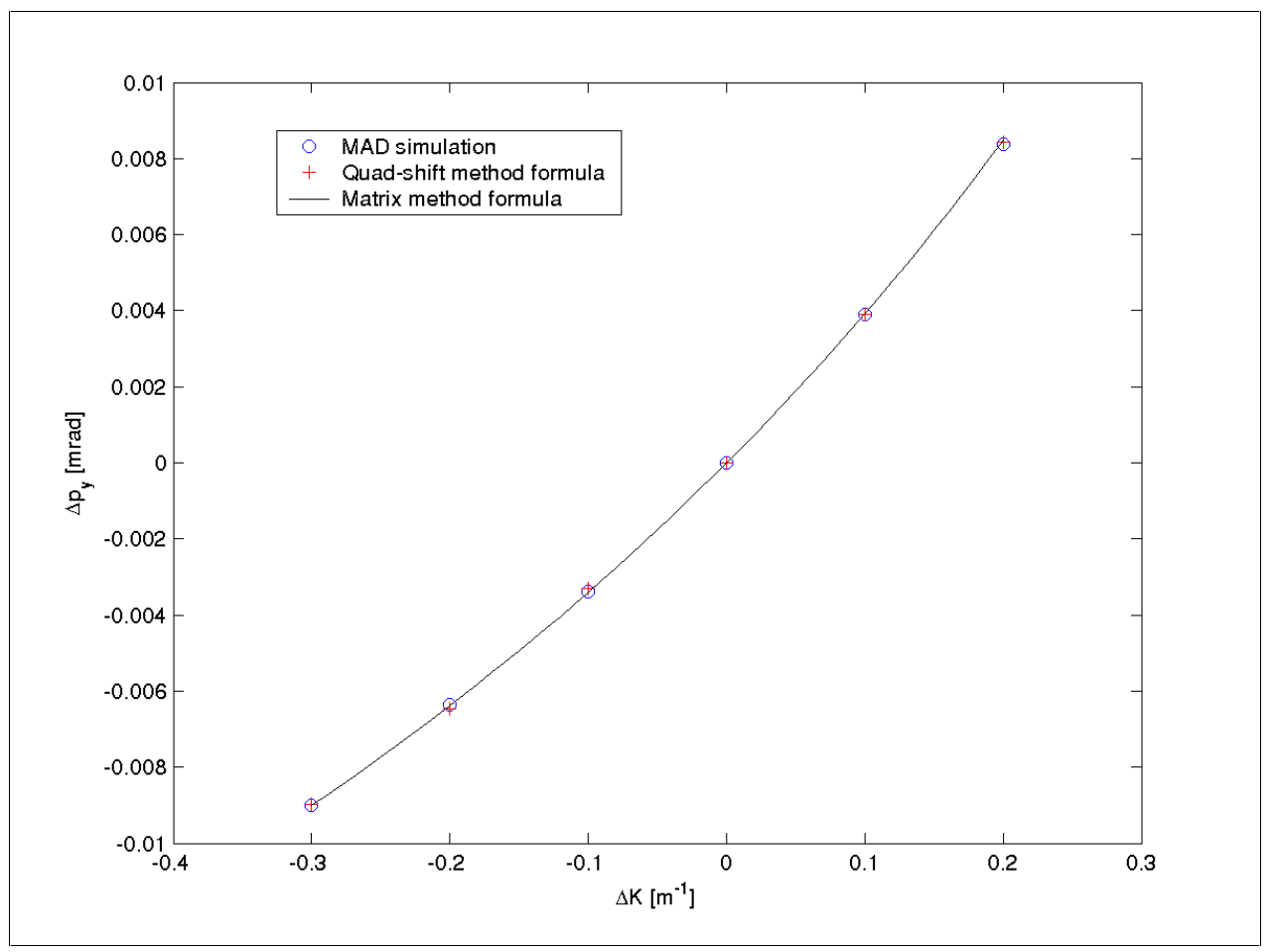

Figure 2

Change of closed orbit angle at QF2R.26 in response to variations in the strength of the quadrupole. 
Table 1

Results of MAD simulation of response of closed orbit to changes in strength of QF2R.26.

\begin{tabular}{|c|c|c|c|c|c|}
\hline $\begin{array}{c}\text { MAD } k_{1} l \\
{\left[\mathrm{~m}^{-1}\right]}\end{array}$ & $\begin{array}{c}\beta_{y} \\
{[\mathrm{~m}]}\end{array}$ & $\alpha_{y}$ & $\begin{array}{c}\text { Vertical Tune } \\
v_{y}\end{array}$ & $\begin{array}{c}y \\
{[\mathrm{~mm}]}\end{array}$ & $\begin{array}{c}p_{y} \\
{[\mathrm{mrad}]}\end{array}$ \\
\hline \hline 1.44225 & 1.28554 & -0.91932 & 8.78887 & 3.55909 & 0.381853 \\
\hline 1.54225 & 1.26799 & -0.97019 & 8.77871 & 3.51666 & 0.377384 \\
\hline 1.64225 & 1.25605 & -1.02387 & 8.76867 & 3.48018 & 0.373486 \\
\hline 1.74225 & 1.24929 & -1.08082 & 8.75871 & 3.4485 & 0.370094 \\
\hline 1.84225 & 1.24746 & -1.14161 & 8.74877 & 3.42074 & 0.367119 \\
\hline 1.94225 & 1.2505 & -1.20691 & 8.73884 & 3.39622 & 0.36449 \\
\hline
\end{tabular}

\subsection{Quadrupole QM14R.2}

One potentially worrisome feature of equations (10) is the possibility for the denominator to be zero, depending on the quadrupole strength, beta function and tune. The condition for zero in the denominator is:

$$
\beta K \cot (\pi v)= \pm 2
$$

where $K$ is the integrated quadrupole strength (positive for a horizontally focusing quadrupole), and the upper sign should be used for the horizontal plane, and the lower sign for the vertical. However, since the equations (10) are the same as equations (6), equations (10) must have a well-defined limit as $\beta K \cot (\pi v) \rightarrow \pm 2$. It appears that equations (6) might also behave badly for particular values of the change in quadrupole strength $\Delta K$; however, we expect the lattice to become unstable, if the quadrupole strength is changed too much, in which case there is indeed no closed orbit.

Although we are likely safe, it is interesting to consider a case for which $\beta K \cot (\pi v) \approx \pm 2$. There are no quadrupoles that exactly satisfy this condition in the ATF lattice in the vertical plane. The quadrupole we considered above, QF2R.26, has $\beta_{y} K \cot \left(\pi v_{y}\right)=2.32$, which is far from -2 . The quadrupole that comes closest to meeting the condition (11) in the vertical plane is QM14R.2 (a quadrupole in one of the straight sections), which has $\beta_{y} K \cot \left(\pi v_{y}\right)=-1.84$. We therefore repeat for QM14R.2, the above modeling and analysis that we carried out for QF2R.26. The results are given in Table 2, and plotted (again compared with the analytical formulae) in Figure 3 and Figure 4.

Not surprisingly, nothing remarkable happens. The quadrupole strength can be varied over a rather narrower range than in the case of QF2R.1. The results of the modeling and the analytical calculations are again in almost exact agreement. 


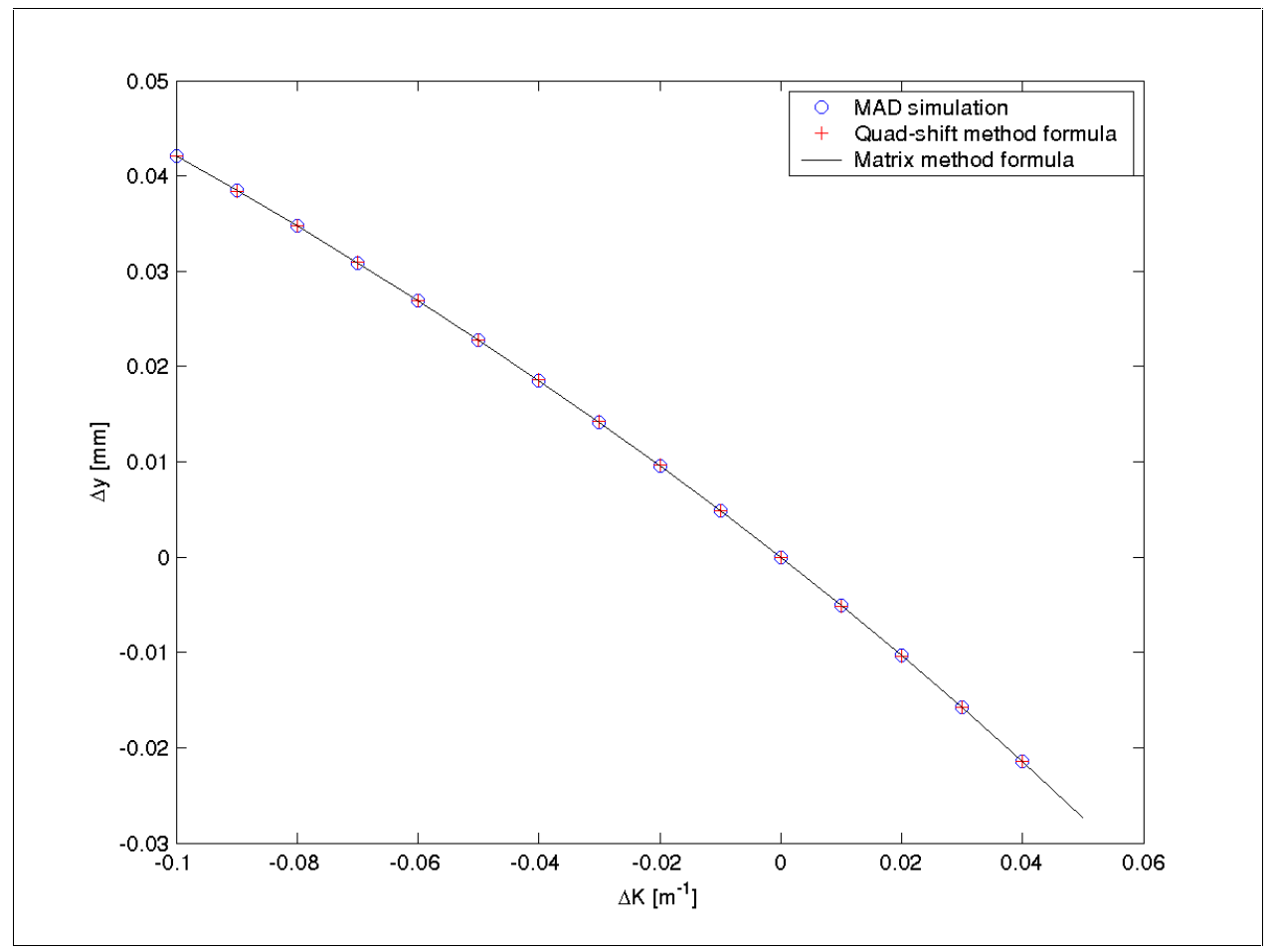

Figure 3

Change of closed orbit co-ordinate at QM14R.2 in response to variations in the strength of the quadrupole.

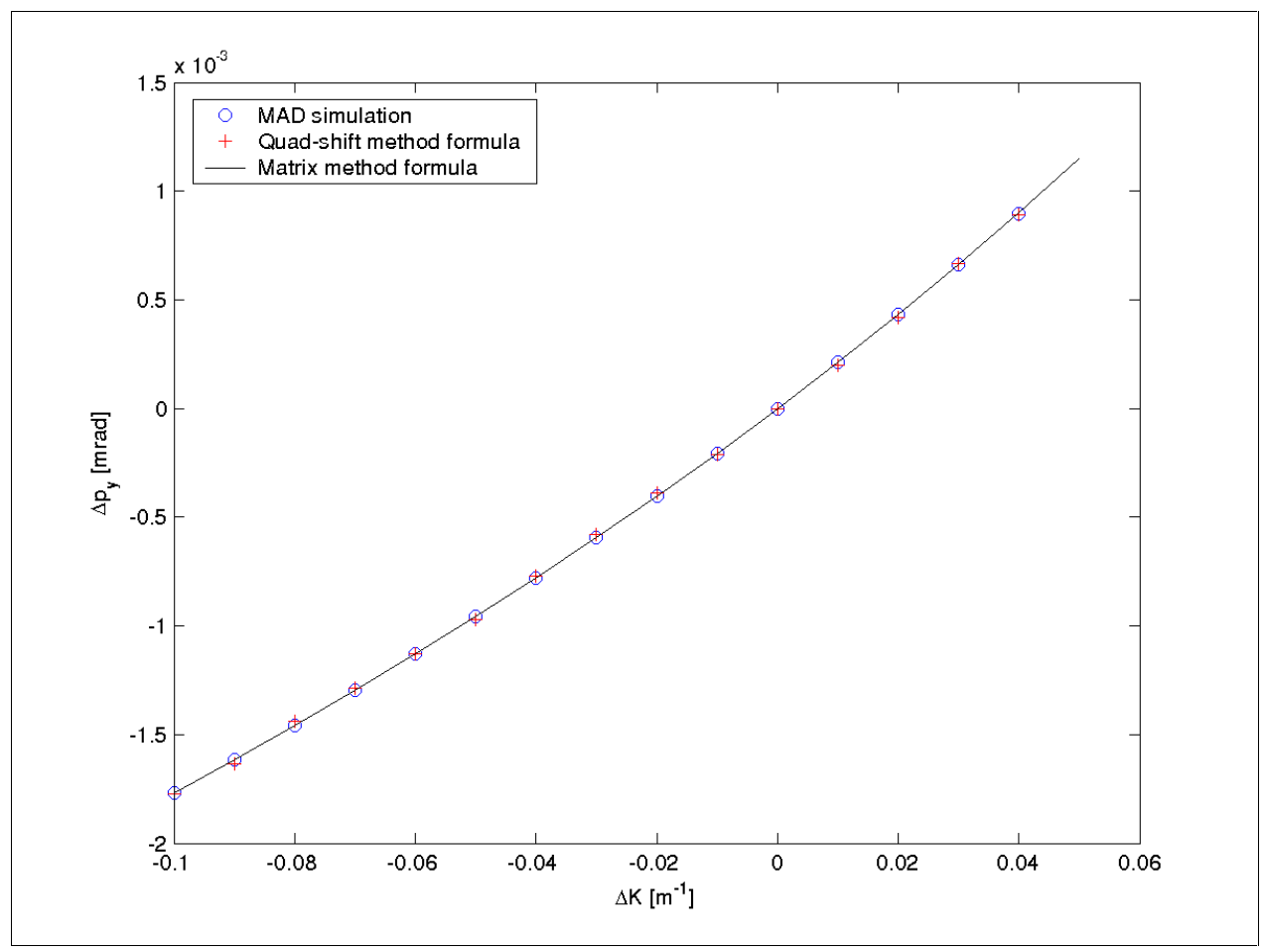

Figure 4

Change of closed orbit angle at QM14R.2 in response to variations in the strength of the quadrupole. 
Table 2

Results of MAD simulation of response of closed orbit to changes in strength of QF2R.26.

\begin{tabular}{|c|c|c|c|c|c|}
\hline $\begin{array}{c}\text { MAD } k_{1} l \\
{\left[\mathrm{~m}^{-1}\right]}\end{array}$ & $\begin{array}{c}\beta_{y} \\
{[\mathrm{~m}]}\end{array}$ & $\alpha_{y}$ & $\begin{array}{c}\text { Vertical Tune } \\
v_{y}\end{array}$ & $\begin{array}{c}y \\
{[\mathrm{~mm}]}\end{array}$ & $\begin{array}{c}p_{y} \\
{[\mathrm{mrad}]}\end{array}$ \\
\hline \hline 0.46278 & 3.22553 & -0.69282 & 8.77981 & 2.70332 & -0.11352 \\
\hline 0.47278 & 3.21613 & -0.70688 & 8.77725 & 2.709 & -0.11376 \\
\hline 0.48278 & 3.20764 & -0.72105 & 8.77469 & 2.71445 & -0.11398 \\
\hline 0.49278 & 3.20003 & -0.73534 & 8.77214 & 2.7197 & -0.11421 \\
\hline 0.50278 & 3.19329 & -0.74976 & 8.7696 & 2.72477 & -0.11442 \\
\hline 0.51278 & 3.1874 & -0.76431 & 8.76706 & 2.72965 & -0.11462 \\
\hline 0.52278 & 3.18235 & -0.77901 & 8.76453 & 2.73437 & -0.11482 \\
\hline 0.53278 & 3.17812 & -0.79387 & 8.762 & 2.73892 & -0.11501 \\
\hline 0.54278 & 3.17472 & -0.80889 & 8.75947 & 2.74332 & -0.1152 \\
\hline 0.55278 & 3.17212 & -0.82409 & 8.75694 & 2.74757 & -0.11537 \\
\hline 0.56278 & 3.17032 & -0.83948 & 8.75442 & 2.75168 & -0.11555 \\
\hline 0.57278 & 3.16932 & -0.85506 & 8.7519 & 2.75567 & -0.11571 \\
\hline 0.58278 & 3.16912 & -0.87085 & 8.74938 & 2.75952 & -0.11588 \\
\hline 0.59278 & 3.16971 & -0.88686 & 8.74685 & 2.76326 & -0.11603 \\
\hline 0.60278 & 3.1711 & -0.90311 & 8.74433 & 2.76688 & -0.11619 \\
\hline
\end{tabular}

\section{Closed Orbit Response to Change in Skew Quadrupole Strengths}

The beam offset in the sextupoles is significant for storage ring operation; the vertical offset, for example, affects the coupling, and is of great importance for damping ring performance. This motivates the need for a beam-based alignment technique that can be applied to the sextupole magnets. At the ATF, all the sextupoles have independently powered skew quadrupole trim windings. This raises the possibility of performing beam-based alignment of the sextupoles, by varying the superposed skew-quadrupole field and observing the change in the closed orbit. Of course, there are many practical considerations; for example, it is not necessarily the case that the magnetic centers of the sextupole and skew quadrupole fields are the same. We do not consider any practical issues here, but simply discuss how the closed orbit in a storage ring varies in response to changes in strength of a skew quadrupole.

We proceed using a method that is a generalization of the quadrupole-shift method, used in Section 2.2 above. This allows us to derive some possibly useful expressions without the need to consider the Twiss parameters, which ought to be dealt with in a manner consistent with fully coupled motion. For brevity, we consider only the change in the co-ordinates of the closed orbit, and not the momenta.

Consider a skew quadrupole with integrated strength $K_{s}$. Shifting the quadrupole a distance $\Delta x_{q}$ horizontally and $\Delta y_{q}$ vertically is physically the same as superposing dipole fields to give horizontal and vertical kicks:

$$
\begin{aligned}
& \theta_{x}=-K_{s} \Delta y_{q} \\
& \theta_{y}=-K_{s} \Delta x_{q}
\end{aligned}
$$


The closed orbit response to such kicks is:

$$
\begin{aligned}
& \Delta x_{c o}(s)=C_{12}\left(s ; s_{0}\right) \theta_{x}+C_{14}\left(s ; s_{0}\right) \theta_{y} \\
& \Delta y_{c o}(s)=C_{32}\left(s ; s_{0}\right) \theta_{x}+C_{34}\left(s ; s_{0}\right) \theta_{y}
\end{aligned}
$$

As before, we look for the distance we have to move the magnet to put the beam exactly through its center:

$$
\begin{aligned}
& \Delta x_{q}=\Delta x_{c o}+x_{b q}=-C_{12}\left(s_{0} ; s_{0}\right) K_{s} \Delta y_{q}-C_{14}\left(s_{0} ; s_{0}\right) K_{s} \Delta x_{q}+x_{b q} \\
& \Delta y_{q}=\Delta y_{c o}+y_{b q}=-C_{32}\left(s_{0} ; s_{0}\right) K_{s} \Delta y_{q}-C_{34}\left(s_{0} ; s_{0}\right) K_{s} \Delta x_{q}+y_{b q}
\end{aligned}
$$

Thus:

$$
\left[1+K_{s} \tilde{C}\left(s_{0} ; s_{0}\right)\right] \cdot\left(\begin{array}{c}
\Delta x_{q} \\
\Delta y_{q}
\end{array}\right)=\left(\begin{array}{l}
x_{b q} \\
y_{b q}
\end{array}\right)
$$

where:

$$
\tilde{C}=\left(\begin{array}{ll}
C_{14} & C_{12} \\
C_{34} & C_{32}
\end{array}\right)
$$

Therefore, the movement of the skew quadrupole needed to put the beam through its center is given by:

$$
\left(\begin{array}{c}
\Delta x_{q} \\
\Delta y_{q}
\end{array}\right)=\left[1+K_{s} \tilde{C}\left(s_{0} ; s_{0}\right)\right]^{-1} \cdot\left(\begin{array}{c}
x_{b q} \\
y_{b q}
\end{array}\right)
$$

As before, we write down the change in the closed orbit resulting from the above movement of the skew quadrupole with its nominal strength, followed by the change resulting from the reverse movement with some different strength $K_{s}^{(1)}$. The net change in the closed orbit at any point in the lattice is:

$$
\left(\begin{array}{l}
\Delta x_{c o} \\
\Delta y_{c o}
\end{array}\right)_{s}=\left[K_{s}^{(1)} \tilde{C}^{(1)}\left(s ; s_{0}\right)-K_{s} \tilde{C}\left(s ; s_{0}\right)\right] \cdot\left[1+K_{s} \tilde{C}\left(s_{0} ; s_{0}\right)\right]^{-1} \cdot\left(\begin{array}{c}
x_{b q} \\
y_{b q}
\end{array}\right)
$$

This is our final result. Notice the similarity in form between equation (12), and the first of equations (9).

\section{Conclusions and Comments}

We have found expressions for the response of the closed orbit in a storage ring to changes in strengths of quadrupoles. Using two different approaches, we found expressions that looked very different; it turns out, however, that the expressions are indeed equivalent. The choice of which expression to use may depend on whether one wishes to calculate the closed orbit response at the quadrupole whose strength is changing, or the closed orbit response at some other point in the lattice. Comparison of the results of calculations using the expressions that we derived with the results of simulations in MAD for two different quadrupoles in the ATF lattice showed almost exact agreement. 
As we mentioned above, this work was motivated by the need to carry out beam-based alignment in the ATF for quadrupoles that do not have a BPM nearby. The ability to calculate the change in closed orbit throughout the lattice in response to the change in strength of a quadrupole suggests the following procedure for steering through the centers of all quadrupoles:

1. Starting from a chosen reference orbit in the machine, vary the strength of one of the quadrupoles, recording the change in orbit at each BPM.

2. Fit the beam offset in the target quadrupole from the measured orbit change.

3. Repeat for each quadrupole in the ring, starting from the same orbit each time.

4. From a calibrated lattice model, generate a response matrix between the orbit corrector magnets and the beam position in the quadrupoles. Invert the matrix, and apply to the measured beam offsets, to determine steering changes for minimizing the beam offset with respect to the quadrupole centers.

Recall that at the ATF, there are a fewer corrector magnets than there are quadrupoles. The matrix inversion in Step 4 will therefore need to be carried out using singular value decomposition, and one can only expect to minimize the beam offset with respect to the quadrupole centers, rather than steer the beam exactly through the centers. A modification of the procedure (using a response matrix between the quadrupole positions and beam position in the quadrupoles) may be used to identify quadrupoles with relatively large misalignments, which may be targeted for physical realignment to improve the performance of the machine.

It will likely be necessary to iterate this procedure, because effects such as coupling, beam offsets in the sextupoles, dispersion etc. will limit the precision with which the beam offsets in the quadrupoles may be measured. In some lattices, for example the ATF, we believe the sextupoles will have a strong effect, and it will be necessary to use either a more general theory than the one we have presented here, or to perform the alignment with reduced (or zero) sextupole strengths.

\section{Acknowledgements}

We should like to thank Marc Ross and Mark Woodley for useful discussions, and the ATF group at KEK for support of beam-based alignment studies on this machine.

\section{References}

[1] D. Robin, G. Portmann, L. Schachinger, "Automated Beam-Based Alignment of the ALS Quadrupoles,"SLAC Internal Report, NLC Note 18 (1995). http://www-project.slac.stanford.edu/lc/local/NLCNotes/NLCnote_18.pdf

[2] G. Portmann, D. Robin, L. Schachinger, "Automated Beam-Based Alignment of the ALS Quadrupoles," LBNL Internal Report LBL-36434 (April 1995), Presented at 16th IEEE Particle Accelerator Conference (PAC 95) and International Conference on High Energy Accelerators (IUPAP), Dallas, Texas, May 1995.

[3] M. G. Minty and F. Zimmermann, "Measurement and Control of Charged Particle Beams," Springer-Verlag (2003).

[4] M. Ross, J. Nelson, M. Woodley, A. Wolski, "Beam-Based Alignment at the KEK Accelerator Test Facility”, Proceedings EPAC 2002. 